\title{
Faire appel au plein potentiel des femmes africaines
}

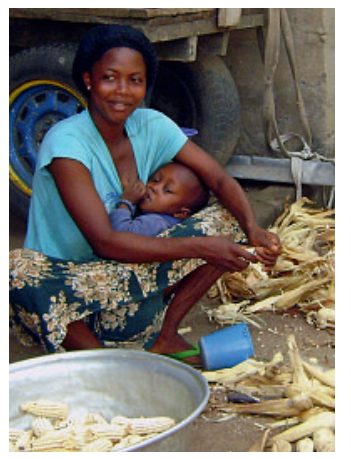

Pour l'essentiel le travail des femmes en Afrique est limité aujourd'hui encore à des tâches liées à la vie quotidienne de la famille.

1 Machel G. La femme est l'avenir de l'Afrique. Le Temps (Genève), 4 juin 2010, p. 13

2 Sur les violences quotidiennes (notamment les viols) à large échelle dans certaines régions, voir les réflexions d'une conseillère nationale au retour de la région des Grands Lacs: Aubert J.

Destins de femmes africaines, 24 Heures (Lausanne) 11 juin 2010, p. 20.

3 A cela il faut ajouter les dégâts liés aux règles coutumières selon quoi la terre ne se transmet que d'homme à homme. Des centaines de milliers de veuves et d'enfants de morts du sida en souffrent. Voir: Martin J. La confiscation des propriétés des veuves et des orphelins Lourd effet collatéral de l'épidémie de VIH/sida. Bull Méd Suisses. 2008;89(45):1955-6.

4 On lira aussi avec intérêt le témoignage d'une jeune Sud-africaine dans le dernier numéro (no 2, juin 2010) de «Un seul monde», revue de la Direction du développement et de la coopération de la Confédération.
Quatre-vingts à cent millions de femmes, essentiellement en Afrique, sont victimes de mutilations génitales féminines (MGF), d'excision sous des formes plus ou moins délabrantes. En Europe, l'excision représente des défis sociaux, médicaux, éthiques, dont les milieux de la gynécologie se préoccupent. La communauté internationale cherche de longue date à agir mais ces pratiques, très ancrées culturellement, ne peuvent être diminuées, et ultimement éradiquées, que par les populations concernées elles-mêmes.

$\mathrm{Au}$ sein des populations, les femmes doivent être les moteurs du changement. Parce que, même si un certain nombre d'hommes voient le côté mutilant et opprimant des MGF, beaucoup restent passifs... parce qu'il est dit que l'excision tend à assurer la fidélité de la femme, diminue sa libido (rappelons qu'elle n'est en rien une prescription religieuse - certains peuples musulmans ne la connaissent pas du tout).

Invitée par Helvetas, organisation de coopération pour qui elle est cheffe de projet au Mali, Mariam Namogo a séjourné en Suisse en mai 2010. Dans son pays, $85 \%$ des femmes sont excisées; trois quarts d'entre elles pensent encore que cette pratique devrait être maintenue. Pourtant, l'excision entraîne chez trois femmes sur dix des conséquences sérieuses (cicatrices vicieuses, incontinence, fistules), sans compter des relations sexuelles difficiles et des complications à l'accouchement susceptibles de se terminer par la mort de l'enfant, voire de la mère. Il existe au Mali un Programme national de lutte contre l'excision mais il peine à changer les mœurs. Contrairement aux pays voisins, le Mali n'a pas encore de loi interdisant l'excision - et, à vrai dire, l'existence d'une loi est assez loin de garantir son application. C'est dire l'importance de l'action de groupes engagés issus de la communauté. Mme Namogo a décrit de manière forte, sage aussi, les efforts qu'elle et ses collègues déploient. Au milieu d'une inertie sociétale, il y a aussi du changement et des motifs d'espérer.

Graça Machel, veuve d'un président du Mozambique et actuelle épouse de Nelson Mandela, est une grande personnalité d'Afrique qui s'exprime dans nos médias [1]: «Ce sont les femmes qui aident à apaiser les tensions et à guérir les terribles blessures dont souffre l'Afrique en raison de la violence. Elles jouent un rôle fondamental dans la résolution des conflits et les pro- cessus de réconciliation» [2]. Les progrès sont surtout insuffisants en ce qui concerne la possibilité pour les femmes d'exploiter pleinement leur talent et de participer à l'économie formelle. Sur le terrain, le véritable moteur de l'économie africaine ce sont elles (le modeste observateur que j'ai été sur place durant quelques années a fait la même constatation), mais elles sont victimes d'injustices de la part du secteur financier par exemple: elles ne reçoivent que $10 \%$ des prêts accordés aux petits agriculteurs, et moins de $1 \%$ des prêts accordés à l'agriculture en général, alors qu'elles ont la responsabilité de $80 \%$ des cultures. Devant ce dernier chiffre on croit rêver mais le fait est que des études solides ont montré qu'une moitié de la population, les femmes, abattent en Afrique les trois quarts du travail - et l'autre moitié, leurs maris, le quart [3]. Mais pour l'essentiel ce travail consiste aujourd'hui encore en tâches liées à la vie quotidienne de la famille, pas assez souvent à des positions d'impulsion et de leadership. Pourtant, écrit Machel, «les femmes investissent et créent des entreprises avec plus de succès que les hommes et respectent mieux les échéances de remboursement». Il y a du progrès toutefois: «Les priorités et les solutions qu'elles proposent ont de plus en plus d'écho, bien que le fossé entre filles et garçons dans l'éducation reste préoccupant (...) Ellen Johnson-Sirleaf est au Libéria la première femme cheffe d'Etat en Afrique, plus de la moitié des parlementaires au Rwanda sont des femmes, des avancées importantes ont été faites en Afrique du Sud et au Lesotho en matière d'égalité» [4].

Ma famille et moi avons vécu en Afrique tropicale et gardons un souvenir chaleureux de la cordialité dans cette partie du monde, des rires éclatants malgré les vicissitudes de la vie. En toute simplicité, j'aimerais dire comment ce que font, à des niveaux très différents, Mariam Namogo et Graça Machel renforce ma conviction que, si on pouvait/voulait bien laisser les femmes y donner leur potentiel, y compris aux plus hauts postes de responsabilité, elles apporteraient une contribution déterminante au développement du continent. Pour cela, il faut «faire tomber les murs qui les empêchent de jouer pleinement leur rôle».

Jean Martin, membre de la rédaction du BMS et de la Commission nationale d'éthique 